\title{
Third-Generation Hydroxyethyl Starch Causes Dose-Dependent Coagulopathy in Patients Undergoing Off-Pump Coronary Artery Bypass with Continuation of Preoperative Aspirin
}

\author{
Go Kusumoto, MD,${ }^{1}$ Midoriko Higashi, $\mathrm{MD}, \mathrm{PhD},{ }^{2}$ Kenji Shigematsu, $\mathrm{MD},{ }^{1}$ Ken Yamaura, $\mathrm{MD}^{2}$ \\ ${ }^{1}$ Department of Anesthesiology, Fukuoka University Hospital, Fukuoka, Japan; \\ ${ }^{2}$ Department of Anesthesiology and Critical Care Medicine, Kyushu University, Fukuoka, Japan
}

\section{ABSTRACT}

Background: We aimed to evaluate the effect of thirdgeneration hydroxyethyl starch (6\% HES 130/0.4) on hemostasis and perioperative blood loss in patients undergoing offpump coronary artery bypass $(\mathrm{OPCAB})$ with continuation of preoperative aspirin.

Methods: Forty-nine consecutive patients, who underwent $\mathrm{OPCAB}$ at a single institution between November 1, 2014 and March 31, 2016, were included. Coagulation tests, including thromboelastometry and clinical data of all patients, retrospectively were collected from anesthesia and medical records.

Results: The total amount of intraoperative crystalloid and HES was $2057.5 \pm 771.6 \mathrm{~mL}(N=32)$ and $1090.6 \pm 645.0$ $\mathrm{mL}(N=32)$, respectively. In the coagulation pathway, the change ratio of fibrinogen concentration, prothrombin time, and fibrinogen thromboelastometry-maximum clot firmness (FIBTEM-MCF) significantly correlated with HES $(P<$ $0.001, P=0.00131$, and $P<0.001$, respectively), but not with crystalloid. In the coagulation pathway concerning interaction with platelets, the change ratio of platelet count, extrinsic thromboelastometry-clotting formation time (EXTEMCFT), and EXTEM-MCF significantly were correlated with HES $(P<0.001, P<0.001$, and $P<0.001$, respectively), but not with crystalloid. At chest closure, the hematocrit decreased in a dose-dependent manner with HES $(P<0.001)$, but not with crystalloid administration. There was an association between the change ratio of hematocrit and EXTEM-MCF $(P$ $=0.00122)$. However, intra-postoperative blood loss was not correlated with HES 130/0.4 or crystalloid administration.

Conclusion: We found that $6 \%$ HES 130/0.4 prolonged coagulation testing in a dose-dependent manner due to hemodilution but did not increase blood loss in patients undergoing $\mathrm{OPCAB}$ with continuation of preoperative aspirin.

Received fune 19, 2021; accepted fuly 14, 2021.

Correspondence: Midoriko Higashi, MD, PbD, Department of Anesthesiology and Critical Care Medicine, Kyushu University, 3-1-1 Maidashi, Higashiku, Fukuoka 812-8582, Japan, Fax +81-92-642-5722 (e-mail: higashi. midoriko.976@m.kyushu-u.ac.jp).

\section{INTRODUCTION}

Optimizing the volume of circulating fluid using colloids reduces postoperative complications [Holte 2006; KozekLangenecker 2013]. However, whether hydroxyethyl starch (HES), including third-generation HES (6\% HES 130/0.4), causes coagulopathy, postoperative bleeding, and acute kidney injury is still controversial [Ahn 2016; Gillies 2014; Min 2017; Navickis 2012; Rasmussen 2016; Schramko 2010; Schramko 2015; Skhirtladze 2014].

Aspirin is beneficial following coronary artery bypass grafting $(\mathrm{CABG})$, and continuation of preoperative aspirin before CABG is widely used in the clinical setting. Although it is related to the risk of postoperative bleeding, it is associated with decreased mortality [Aboul-Hassan 2017]. Further, the effects of HES 103/0.4 on blood loss and coagulation in patients with recent exposure to dual antiplatelet therapy undergoing off-pump CABG (OPCAB) are reportedly lower [Lee 2011]. However, the effect of 6\% HES 130/0.4 on patient hemostasis while undergoing CABG with aspirin continuation is not well known.

Therefore, we aimed to evaluate the effects of $6 \%$ HES $130 / 0.4$ on hemostasis and perioperative blood loss in patients undergoing $\mathrm{OPCAB}$ with continuation of preoperative aspirin.

\section{MATERIALS AND METHODS}

Study population: This retrospective study was approved by an Institutional Ethics Committee/Review Board (IRB No. 15-10-14). We enrolled patients who underwent primary OPCAB with a single surgeon at one institution between November 1, 2014 and March 31, 2016. We excluded patients who underwent concomitant valve surgery, aortic surgery, or conversion to cardiopulmonary bypass (CPB).

Baseline characteristics: Clinical data for all patients, including demographic data, laboratory data, medication use, perioperative management, and postoperative outcomes, retrospectively were collected from anesthesia and medical records.

Anesthetic management: General anesthesia was induced using propofol, fentanyl, and/or continuous infusion of remifentanil and rocuronium, and maintained using propofol or sevoflurane, fentanyl, and continuous infusion of 
remifentanil. In addition to standard monitoring, we monitored direct arterial pressure through a radial artery catheter, central venous pressure, pulmonary artery pressure, and mixed venous oxygen saturation using a pulmonary arterial catheter. After anesthesia induction, all patients underwent continuous intravenous infusion of nitroglycerin and diltiazem. When necessary, ephedrine, phenylephrine, dopamine, dobutamine, norepinephrine, or phosphodiesterase III inhibitors were administered. The fluids infused were chosen by considering hemodynamic parameters, such as blood pressure, heart rate, pulmonary arterial pressure, and transesophageal echocardiography findings. A cell salvage device was used for all patients.

Each anesthesiologist independently decided whether to administer crystalloids (acetate Ringer's solution) or colloids (6\% HES 130/0.4: Voluven, Fresenius Kabi, Bad Homburg, Germany). Six percent HES 130/0.4 was administered only during surgery and not in the intensive care unit (ICU). Red blood cell (RBC) concentrates mainly were transfused at hemoglobin 7-9 g/dL and hematocrit 24\% [Chassot 2004; Windecker 2014]. Intraoperative transfusion of fresh-frozen plasma (FFP) and platelets was performed based on each patient's coagulation profile, continuous microvascular bleeding, and standard coagulation test findings, including fibrinogen level.

Heparin was administered at a dose of $100 \mathrm{IU} / \mathrm{kg}$ to achieve a target activated clotting time (ACT) between 200 $\mathrm{s}$ and $300 \mathrm{~s}$ after dissection of the internal mammary artery, radial artery, and/or saphenous vein grafts. ACT assessments were repeated every $60 \mathrm{~min}$, with heparin added as required. After completion of all anastomoses, protamine was administered at $1 \mathrm{mg} /$ heparin $100 \mathrm{IU}$, and added as required, to return the ACT to preoperative levels. After surgery, all patients were transferred to the ICU. Based on surgeon's discretion, they conducted postoperative management, which included fluid therapy, transfusion, mechanical ventilation, and hemodynamic assessment. Postoperative blood loss, such as that by chest tube drainage and transfusion, was recorded $24 \mathrm{~h}$ after surgery.

Thromboelastometry and laboratory analyses: We routinely collected arterial blood samples from the radial artery by anesthesia induction before heparin administration, after protamine administration, at chest closure, and in the ICU. Hemoglobin, hematocrit, platelets, ACT, prothrombin time (PT), international normalized ratio of prothrombin time (PT-INR), activated partial thromboplastin time (aPTT), fibrinogen level, and rotational thromboelastometry (ROTEM ${ }^{\circledR}$ Tem Innovations $\mathrm{GmbH} \mathrm{CO}$, Munich, Germany) parameters were measured using the obtained samples. We performed extrinsic thromboelastometry (EXTEM) and fibrinogen thromboelastometry (FIBTEM) tests using ROTEM® immediately after obtaining the blood samples. EXTEM was performed to test the extrinsic coagulation pathway with respect to the interaction with platelets. FIBTEM was performed to test the quality of fibrin polymerization and fibrinogen concentration. Coagulation was allowed for $\geq 30$ min. Each attending anesthesiologist did not use ROTEM ${ }^{\circledR}$ data as coagulation management.
Outcome variables and statistical analysis: The primary outcome variable was the effect of the fluid volume administered from anesthesia induction to chest closure on hemostasis parameters evaluated by standard coagulation monitoring and ROTEM®. The effect of fluids on hemostasis was evaluated based on the changes observed from baseline. The secondary outcomes were the effect of the fluid volume administered during surgery on intraoperative and postoperative blood loss and the postoperative change of estimated glomerular filtration rate (eGFR).

Data values are expressed as mean \pm standard deviation or number. Based on the data distribution, Pearson's correlation coefficient test and Spearman rank correlation test were used to test the correlation coefficient between intraoperative and postoperative blood loss, laboratory hemostatic variables, and ROTEM® parameters. Correlation was defined when the correlation coefficient was $>0.4$ [Schober 2018]. A two-sided P-value $<0.05$ was regarded as statistically significant. Because this was a retrospective study using exploratory and descriptive analyses, no sample size calculation was performed. Data were analyzed using EZR (Saitama Medical Center, Jichi Medical University, Saitama Japan [Kanda 2013]), which is a graphical user interface for $\mathrm{R}$ (The R Foundation for Statistical Computing, Vienna, Austria). More precisely, it is a modified version of $\mathrm{R}$ commander designed to add statistical functions frequently used in biostatistics.

\section{RESULTS}

A total of 49 patients underwent OPCAB, during the study period. After excluding patients who converted to $\mathrm{CPB}(N=$ 8), patients with missing important perioperative data $(N=$ $8)$, and patients who did not take aspirin preoperatively ( $N$ =1), we included data from 32 patients (Figure 1). Patient characteristics and perioperative data are shown in Table 1. All patients received aspirin, and two patients used heparin preoperatively.

The total volume of crystalloids and HES 130/0.4 administered intraoperatively was $2057.5 \pm 771.6 \mathrm{~mL}(N=32)$ and $1090.6 \pm 645.0 \mathrm{~mL}(N=32)$, respectively. In the coagulation pathway, the change ratio of the fibrinogen concentration and FIBTEM-maximum clot firmness (MCF) was significantly reduced with HES 130/0.4, but not with crystalloid administration $(P<0.001$, correlation coefficient $=-0.614$ and $P<0.001$, correlation coefficient $=-0.608$, respectively) (Figure 2). The PT change ratio was significantly prolonged with HES 130/0.4, but not with crystalloid administration $(P$ $=0.00131$, correlation coefficient $=0.543)($ Figure 2). The aPTT and ACT change ratios were not significantly correlated with HES 130/0.4 or crystalloid administration.

In the coagulation pathway, the platelet count change ratio significantly decreased with HES 130/0.4, but not with crystalloid administration $(P<0.001$, correlation coefficient $=-0.726)$ (Figure 3). The change ratio of the EXTEM clotting formation time (CFT) and EXTEM-MCF significantly was longer with HES 130/0.4, but not with crystalloid, in a dose-dependent manner $(P<0.001$, correlation coefficient $=0.684$ and $P<0.001$, correlation coefficient $=-0.722$, respectively) $($ Figure 3$)$. 


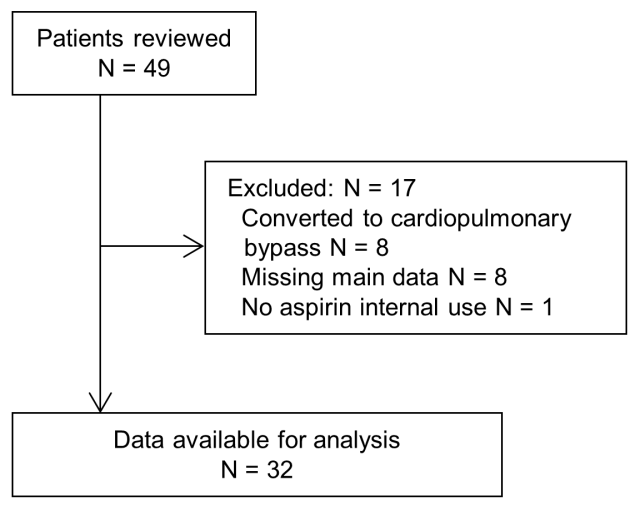

Figure 1. Flow diagram of the study inclusion/exclusion process. A total of 49 patients underwent off-pump coronary artery bypass surgery (OPCAB) during the study period. After excluding patients who converted to cardiopulmonary bypass $(N=8)$, patients missing important perioperative data $(N=8)$, and patients who did not take aspirin preoperatively $(N=1)$, we included data from the remaining 32 patients in the final analysis.

Table 1. Patient characteristics and perioperative data

\begin{tabular}{ll}
\hline Age $(\mathrm{yr})$ & $71.9 \pm 9.2$ \\
Gender (male/female) & $27 / 5$ \\
Height (cm) & $160.1 \pm 8.9$ \\
Weight $(\mathrm{kg})$ & $61.5 \pm 10.6$ \\
$\mathrm{BMI}(\mathrm{kg} / \mathrm{m} 2)$ & $24.0 \pm 3.9$ \\
$\mathrm{NYHA}(\mathrm{I} / \mathrm{II} / \mathrm{III} / \mathrm{IV})$ & $13 / 17 / 2 / 0$ \\
ASA $(1 / 2 / 3 / 4 / 5)$ & $0 / 12 / 19 / 1 / 0$ \\
Preoperative use of aspirin (n) (\%) & $32(100)$ \\
Preoperative use of heparin (n) & 2 \\
Intraoperative use of heparin (units) & $7725.8 \pm 2020.7$ \\
Protamine (mg) & $57.4 \pm 14.4$ \\
Re-exploration for bleeding (n) & 0 \\
Time to extubation (h) & $32.7 \pm 44.2$ \\
ICU stay (h) & $64.4 \pm 44.1$ \\
Mortality (1M) & 0 \\
\hline
\end{tabular}

Data are expressed as number of patients or mean \pm standard deviation. BMI, body mass index; NYHA, New York Heart Association functional classification; ASA, American Society of Anesthesiologists physical status classification; ICU, intensive care unit

The hematocrit decreased with HES 130/0.4 administration in a dose-dependent manner $(P<0.001$, correlation coefficient $=-0.649)$ at chest closure, but not with crystalloid administration (Figure 4). There was an association between the hematocrit change ratio and EXTEM-MCF $(P=0.00122$, correlation coefficient $=0.546)($ Figure 4$)$.

The amount of intraoperative bleeding was $330.9 \pm 187.0$ $\mathrm{mL}$, and that within $24 \mathrm{~h}$ after surgery was $643.4 \pm 259.6 \mathrm{~mL}$.
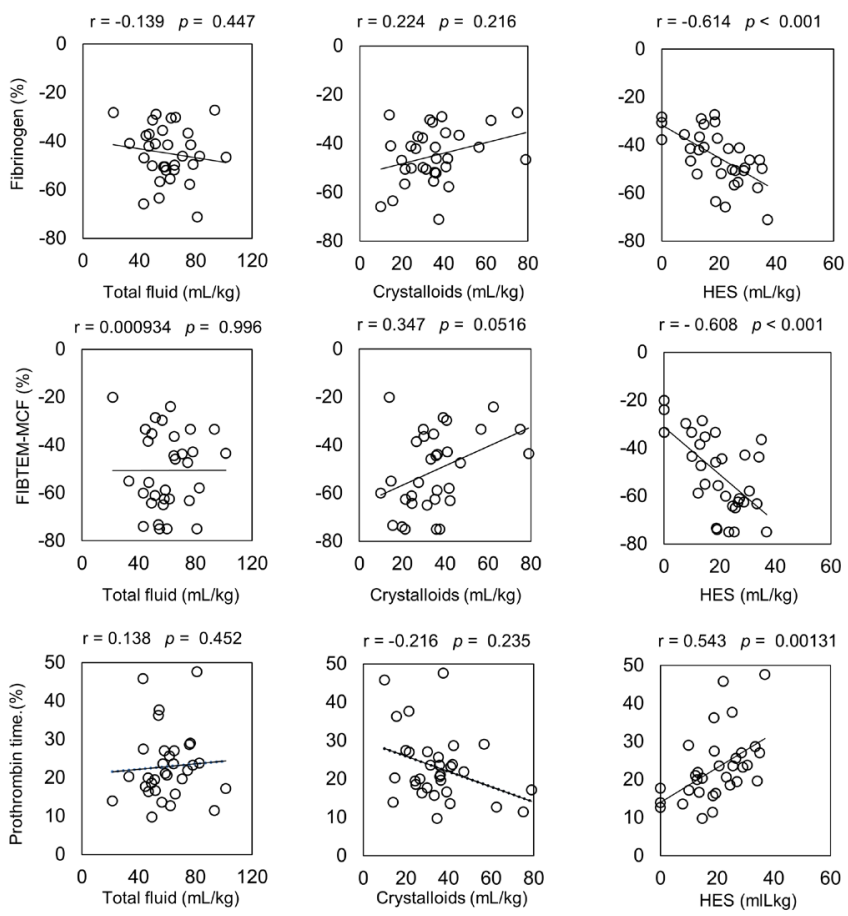

Figure 2. Change in coagulation test results following fluid administration from anesthesia induction to chest closure. Hydroxyethyl starch (HES) $130 / 0.4$, but not crystalloid, administration was significantly correlated with a decrease in fibrinogen concentration $(P<0.001$, correlation coefficient $=-0.614)$, fibrinogen thromboelastometry-maximum clot firmness (FIBTEM-MCF) $(P<0.001$, correlation coefficient $=-0.608)$, and prothrombin time $(P T)(P=0.00131$, correlation coefficient $=0.543)$.

The RBC, FFP, and platelet transfusion requirements during surgery and those within $24 \mathrm{~h}$ after surgery were $3.8 \pm 2.9$ units, $2.9 \pm 5.1$ units, and none and $1.4 \pm 1.8$ units, $2.5 \pm 3.4$ units, and $0.3 \pm 1.8$ units, respectively. Blood transfusion and blood loss did not correlate with HES 130/0.4 or crystalloid administration (data not shown).

The change of eGFR in the patients $(N=28)$, excluding patients who were dependent on hemodialysis $(N=4)$, was from $55.23 \pm 18.32 \mathrm{~mL} / \mathrm{min} / 1.73 \mathrm{~m} 2$ before surgery to 51.49 $\pm 21.5 \mathrm{~mL} / \mathrm{min} / 1.73 \mathrm{~m} 2$ at postoperative day 1 . The eGFR was not decreased with HES $130 / 0.4$ administration $(P=$ 0.34 , correlation coefficient $=0.187$ ) .

\section{DISCUSSION}

In the present study, HES 130/0.4 administration in patients undergoing $\mathrm{OPCAB}$ with continuation of preoperative aspirin in a dose-dependent manner affected coagulation parameters concomitant with platelets, such as platelet count, EXTEMCFT, and EXTEM-MCF, and platelet-independent coagulation parameters, such as fibrinogen concentration, FIBTEMMCF, and PT, but did not affect the amount of blood loss.

Direct and indirect effects could be responsible for the reduction in coagulation parameters due to HES 130/0.4. 

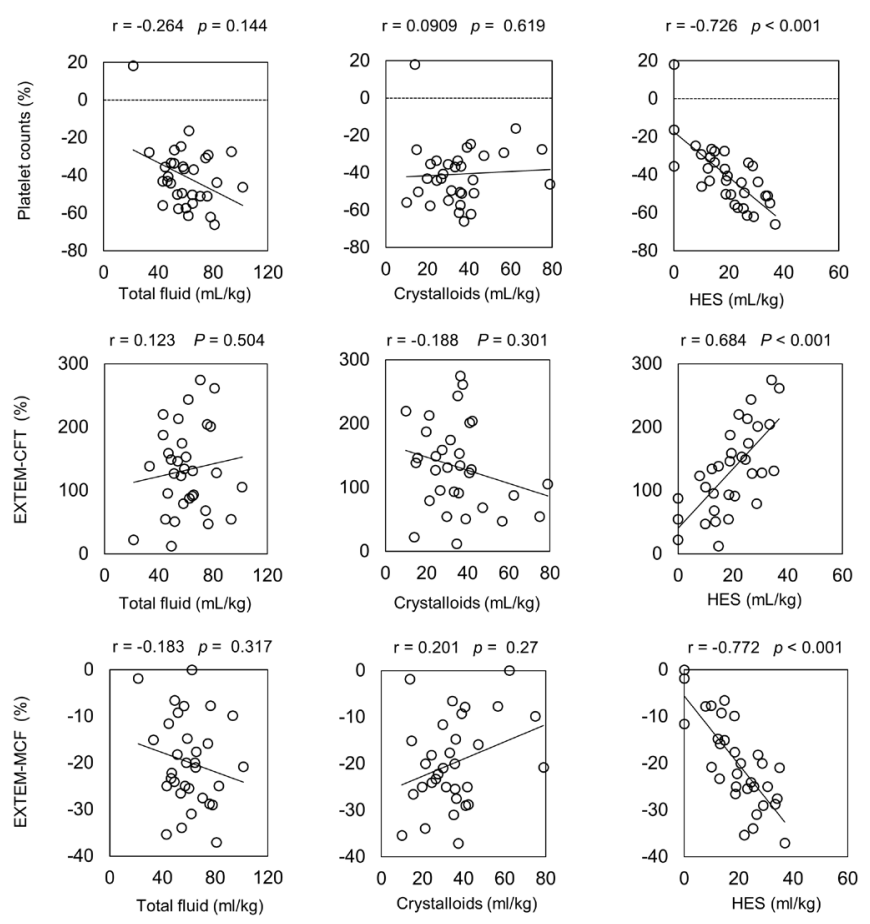

Figure 3. Change in the coagulation pathway concerning interaction with platelets following fluid administration from anesthesia induction to chest closure. The change ratio of platelet count, extrinsic thromboelastometry-clotting formation time (EXTEM-CFT), and EXTEM-maximum clot firmness (MCF) significantly correlated in a dose-dependent manner with hydroxyethyl starch (HES) $130 / 0.4$, but not with crystalloid administration $(P=<0.001$, correlation coefficient $=-0.726 ; P=<$ 0.001 , correlation coefficient $=0.684 ; P=<0.001$, correlation coefficient $=-0.722$, respectively).

Decreased FIBTEM-MCF indicates an impairment of fibrin polymerization, which is a direct effect of HES. This is consistent with previous reports that fibrinogen and/or factor VIII administration did not improve [Kind 2013; Schlimp 2013]. On the other hand, the reason why aPTT was not prolonged is thought to be that HES 130/0.4 is rapidly degraded. Prolonged aPTT is due to decrease factor VIII by slowdegrading type HES [Kozek-Langenecker 2005; Kozek-Langenecker 2008].

There are concerns about HES 130/0.4 increasing the risk of bleeding in patients with continued antiplatelet therapy in the perioperative period, and HES also is known to result in impaired platelet function, in a molecular weight-dependent pattern; this impairment is greater with HES 200 than with HES 130 [Kozek-Langenecker 2008; Liu 2009]. Accordingly, a modified thromboelastography analysis in patients with recent exposure to dual antiplatelet therapy undergoing OPCAB found a lower effect of HES 130/0.4 $(\leq 30 \mathrm{~mL} / \mathrm{kg})$ on platelet function [Lee 2011]. However, in the present study, HES 130/0.4 decreased platelet counts and EXTEM-MCF and prolonged EXTEM-CFT (time from CT to $20 \mathrm{~mm}$ of amplitude), which is a marker of clot formation speed in relation to platelet function. HES decreased the von Willebrand
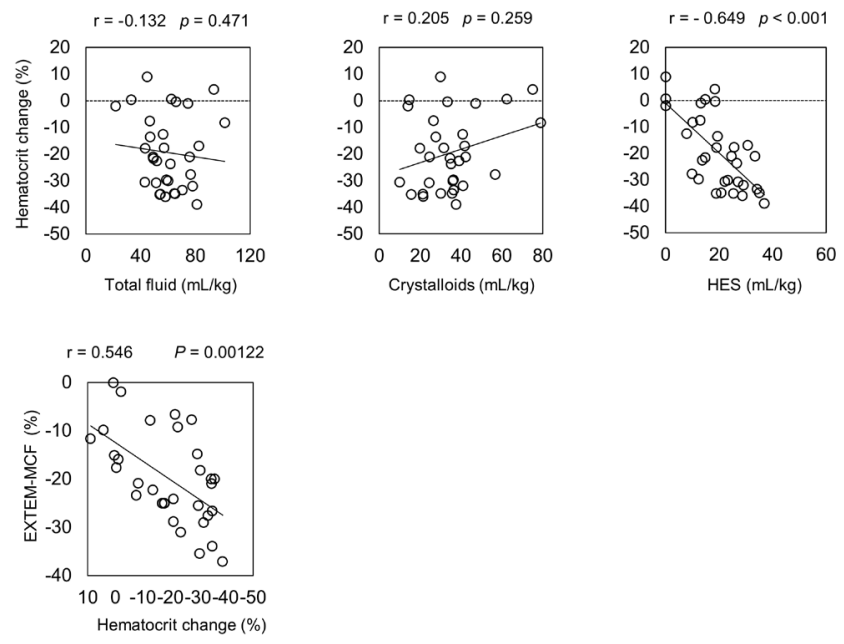

\begin{abstract}
Figure 4. Correlation between fluid administration and hematocrit level change and extrinsic thromboelastometry-maximum clot firmness and hematocrit level change from anesthesia induction to chest closure. Hydroxyethyl starch (HES) 130/0.4, but not crystalloid, dose-dependently decreased hematocrit levels at chest closure $(P<0.001$, correlation coefficient $=-0.649)$. There was an association between the change ratio of the extrinsic thromboelastometry-maximum clot firmness (EXTEMMCF) and hematocrit and total fluid during the intraoperative period ( $P$ $=0.00122$, correlation coefficient $=0.546$ ).
\end{abstract}

factor levels and platelet-related protein and phosphatidylserine expression in a dose-dependent manner but did not affect GP IIb/IIIa. Thus, the extrinsic coagulation pathway effects on platelet interaction were impaired in a dose-dependent manner by HES 130/0.4 [Jin 2017; Kozek-Langenecker 2005; Reuteler 2017].

In the present study, the hematocrit was significantly and dose-dependently decreased after HES 130/0.4 administration, suggesting that colloid administration induces hemodilution. This hemodilution could cause a decrease in platelet count and function and fibrinogen concentration, thus resulting in a decrease in EXTEM-MCF and prolonged PT. Hemodilution is considered the main cause of HES-induced coagulopathy [Schlimp 2013; Skhirtladze 2014]. If the hemodilution effect of HES 130/0.4 is the same as that of HES $200 / 0.5$ or crystalloids, blood loss and transfusion requirements during coronary artery bypass grafting would not differ [Kasper 2003; Lee 2011]. HES-induced hemodilution interferes with phosphatidylserine combination with factor XIa, factor VIIa, and factor Va, which affect the acceleration and explosion stage of thrombin [Jin 2017]. Our results showing that decreased hematocrit was related to decreased EXTEM-MCF also indicated that hemodilution is the main cause of coagulopathy.

The effects of $6 \%$ HES 130/0.4 administration on blood loss remain controversial [Min 2017; Rasmussen 2014; Rasmussen 2016; Skhirtladze 2014]. The increase in blood loss in cardiac surgery is greater with slowly degradable HES than with rapidly degradable $6 \%$ HES $130 / 0.4$. As shown in our study, $6 \%$ HES 130/0.4 had no significant effect on bleeding and transfusion [Gallandat 2000; Kasper 2003; Min 2017; 
Rasmussen 2016; Skhirtladze 2014; Wilkes 2001]. In line with our results, previous reports found similar effects of HES $130 / 0.4$ on blood loss even in patients receiving antiplatelet therapy [Lee 2011].

The present study has several limitations. First, this was a retrospective study with a small sample size. In this study, to reduce the influence of the surgeon, we limited our data to surgeries from a single surgeon. Second, perioperative management was not controlled with anesthesia, and postoperative management was performed by several anesthesiologists and surgeons. Therefore, after determining the criteria for blood transfusion, it is necessary to compare them in prospective study.

\section{CONCLUSION}

We found that $6 \%$ HES 130/0.4 led to prolonged coagulation testing in a dose-dependent manner to hemodilution but did not increase blood loss in patients undergoing CABG with continuation of preoperative aspirin.

\section{ACKNOWLEDGEMENT}

Ethical approval: This retrospective study was approved by the Institutional Ethics Committee/Review Board (IRB No. 15-10-14). The need for informed consent was waived due to the retrospective design.

\section{REFERENCES}

Aboul-Hassan SS, Stankowski T, Marczak J, et al. 2017. The use of preoperative aspirin in cardiac surgery: A systematic review and meta-analysis. J Card Surg. 32:758-74.

Ahn HJ, Kim JA, Lee AR, et al. 2016. The Risk of Acute Kidney Injury from Fluid Restriction and Hydroxyethyl Starch in Thoracic Surgery. Anesth Analg. 122:186-93.

Chassot PG, van der Linden P, Zaugg M, Mueller XM, Spahn DR. 2004. Off-pump coronary artery bypass surgery: physiology and anaesthetic management. Br J Anaesth. 92:400-13.

Gallandat Huet RC, Siemons AW, Baus D, et al. 2000. A novel hydroxyethyl starch (Voluven) for effective perioperative plasma volume substitution in cardiac surgery. Can J Anaesth. 47:1207-15.

Gillies MA, Habicher M, Jhanji S, et al. 2014. Incidence of postoperative death and acute kidney injury associated with i.v. $6 \%$ hydroxyethyl starch use: systematic review and meta-analysis. Br J Anaesth. 112:25-34.

Holte K, Kehlet H. 2006. Fluid therapy and surgical outcomes in elective surgery: a need for reassessment in fast-track surgery. J Am Coll Surg. 202:971-89.

Jin S, Yu G, Hou R, Shen B, Jiang H. 2017. Effect of Hemodilution In Vitro with Hydroxyethyl Starch on Hemostasis. Med Sci Monit. 23:2189-97.

Kanda Y. 2013. Investigation of the freely available easy-to-use software 'EZR' for medical statistics. Bone Marrow Transplant. 48:452-8.

Kasper SM, Meinert P, Kampe S, et al. 2003. Large-dose hydroxyethyl starch 130/0.4 does not increase blood loss and transfusion requirements in coronary artery bypass surgery compared with hydroxyethyl starch 200/0.5 at recommended doses. Anesthesiology. 99:42-7.

Kind SL, Spahn-Nett GH, Emmert MY, et al. 2013. Is dilutional coagulopathy induced by different colloids reversible by replacement of fibrinogen and factor XIII concentrates? Anesth Analg. 117:1063-71.

Kozek-Langenecker SA. 2005. Effects of hydroxyethyl starch solutions on hemostasis. Anesthesiology. 103:654-60

Kozek-Langenecker SA, Afshari A, Albaladejo P, et al. 2013. Management of severe perioperative bleeding: guidelines from the European Society of Anaesthesiology. Eur J Anaesthesiol. 30:270-382.

Kozek-Langenecker SA, Jungheinrich C, Sauermann W, Van der Linden P. 2008. The effects of hydroxyethyl starch 130/0.4 (6\%) on blood loss and use of blood products in major surgery: a pooled analysis of randomized clinical trials. Anesth Analg. 107:382-90.

Lee JS, Ahn SW, Song JW, et al. 2011. Effect of hydroxyethyl starch $130 / 0.4$ on blood loss and coagulation in patients with recent exposure to dual antiplatelet therapy undergoing off-pump coronary artery bypass graft surgery. Circ J. 75:2397-402.

Liu FC, Liao CH, Chang YW, Liou JT, Day YJ. 2009. Hydroxyethyl starch interferes with human blood ex vivo coagulation, platelet function and sedimentation. Acta Anaesthesiol Taiwan. 47:71-8.

Min JJ, Cho HS, Jeon S, et al. 2017. Effects of 6\% hydroxyethyl starch $130 / 0.4$ on postoperative blood loss and kidney injury in off-pump coronary arterial bypass grafting: A retrospective study. Medicine (Baltimore). 96:e6801.

Navickis RJ, Haynes GR, Wilkes MM. 2012. Effect of hydroxyethyl starch on bleeding after cardiopulmonary bypass: a meta-analysis of randomized trials. J Thorac Cardiovasc Surg. 144:223-30.

Rasmussen KC, Johansson PI, Hojskov M, et al. 2014. Hydroxyethyl starch reduces coagulation competence and increases blood loss during major surgery: results from a randomized controlled trial. Ann Surg. $259: 249-54$

Rasmussen KC, Secher NH, Pedersen T. 2016. Effect of perioperative crystalloid or colloid fluid therapy on hemorrhage, coagulation competence, and outcome: A systematic review and stratified meta-analysis. Medicine (Baltimore). 95:e4498.

Reuteler A, Axiak-Flammer S, Howard J, Adamik KN. 2017. Comparison of the effects of a balanced crystalloid-based and a saline-based tetrastarch solution on canine whole blood coagulation and platelet function. J Vet Emerg Crit Care (San Antonio). 27:23-34.

Schlimp CJ, Cadamuro J, Solomon C, Redl H, Schochl H. 2013. The effect of fibrinogen concentrate and factor XIII on thromboelastometry in $33 \%$ diluted blood with albumin, gelatine, hydroxyethyl starch or saline in vitro. Blood Transfus. 11:510-7.

Schober P, Boer C, Schwarte LA. 2018. Correlation coefficients: appropriate use and interpretation. Anesth Analg 126:1763-8.

Schramko A, Suojaranta-Ylinen R, Kuitunen A, et al. 2010. Hydroxyethylstarch and gelatin solutions impair blood coagulation after cardiac surgery: a prospective randomized trial. Br J Anaesth. 104:691-7.

Schramko A, Suojaranta-Ylinen R, Niemi T, et al. 2015. The use of balanced HES 130/0.42 during complex cardiac surgery; effect on blood coagulation and fluid balance: a randomized controlled trial. Perfusion. 30:224-32

Skhirtladze K, Base EM, Lassnigg A, et al. 2014. Comparison of the effects of albumin 5\%, hydroxyethyl starch 130/0.4 6\%, and Ringer's 
lactate on blood loss and coagulation after cardiac surgery. Br J Anaesth. 112:255-64.

Wilkes MM, Navickis RJ, Sibbald WJ. 2001. Albumin versus hydroxyethyl starch in cardiopulmonary bypass surgery: a meta-analysis of postoperative bleeding. Ann Thorac Surg 72:527-33. discussion 534.
Windecker S, Kolh P, Alfonso F, et al. 2014. ESC/EACTS Guidelines on myocardial revascularization: The Task Force on Myocardial Revascularization of the European Society of Cardiology (ESC) and the European Association for Cardio-Thoracic Surgery (EACTS)Developed with the special contribution of the European Association of Percutaneous Cardiovascular Interventions (EAPCI). Eur Heart J. 35:2541-619. 